\title{
MTRA - Status Quo und Weg in die Zukunft
}

\begin{abstract}
Rasante Entwicklungen in Technologie und Wissenschaft, aber auch der demografische Wandel und der damit einhergehende Fachkräftemangel stellen für den Beruf des Medizinisch-TechnischenRadiologie-Assistenten große Herausforderungen dar. Welche konkreten Anforderungen sich daraus - auch mit Blick auf die in Deutschland immer noch gültige Ausbildungs- und Prüfungsverordnung von 1994 - ergeben, erläutern Anke Ohmstede und Katja Röhr in einem gemeinsamen Gespräch.
\end{abstract}

? „Suche MTRA oder MFA mit Röntgenschein" - Stellenanzeigen wie diese finden sich häufig. Sind Medizinisch-Technische-RadiologieAssistenten vom Aussterben bedroht beziehungsweise perspektivisch ersetzbar?

Röhr: Vom Aussterben bedroht sind MTRA sicher nicht, aber man kann schon von einer Art Mangelware sprechen. Leider ist es immer noch so, dass die berufliche Ausbildung zur Medizinisch-Technischen-Radiologie-Assistentin beziehungsweise Assistent bei Jugendlichen zu wenig bekannt ist. Hinzu kommt, dass es für die in der Ausbildungs- und Prüfungsverordnung gesetzlich verankerte 3-jährige Berufsfachschulausbildung keine Ausbildungsvergütung gibt. Dies hat zur Folge, dass immer weniger junge Menschen diesen Beruf ergreifen wollen. Radiologen müssen deshalb notgedrungen auf MFA zurückgreifen, weil sie die vakanten Stellen nicht mit ausgebildeten MTRA besetzen können.

Ohmstede: Es hat natürlich auch eine gewisse Tradition, dass MTRA, MFA oder früher die Arzthelferin in demselben Berufsfeld tätig sind. In meiner über 30-jährigen Berufstätigkeit hat es Arzthelferinnen oder später MFA mit Röntgenschein gegeben, die auch in radiologischen Praxen tätig waren, allerdings zum überwiegenden Teil bei Teilgebietsradiologen, insbesondere bei Orthopäden und Chirurgen. Wenn man sich die Statistiken anschaut, kann man eine wellenförmige Entwicklung beim Nachwuchs und damit auch bei der Auslastung von Ausbildungs- und Arbeitsplätzen erkennen. Neu ist jedoch die
Expansion in der Radiologie gerade in den letzten 10 bis 15 Jahren. Fast jedes Krankenhaus hat ein CT und mehrere MRT. Hinzu kommen im ambulanten Bereich zahlreiche große Verbünde von radiologischen Praxen sowie eine Zunahme niedergelassener Strahlentherapeuten. Nicht zu vergessen das Mammografie-Screening, das in diesem Jahr sein 10-jähriges Bestehen feiert. Allein hier arbeiten rund 2000 MTRA, die damit dem allgemeinen Arbeitsmarkt gar nicht mehr zur Verfügung stehen. Es ist schon erstaunlich, dass vor diesem Hintergrund bislang keine aktivere Arbeitsmarktpolitik betrieben wurde.

? Wie unterscheiden sich denn die Arbeitsfelder eines / r MTRA heute zu denen von vor 15 Jahren?

Röhr: Neu ist sicherlich das schon angesprochene flächendeckende Mammografie-Screening. Aber auch die zahlreichen neuen Großgeräte wie z.B. PET/CT und PET/MR ziehen neue Arbeitsanforderungen und -bedarfe nach sich.

Ohmstede: Genau. Gerade in der Nuklearmedizin haben wir eine Geräteentwicklung, die es vor 15 Jahren in diesem Ausmaß noch nicht gab. Nehmen Sie zum Beispiel die Hybrid-Geräte, auch wenn diese noch nicht so weit verbreitet sind. Im Gegensatz zu vielen anderen technischen Bereichen, wo Technisierung unter Umständen den Wegfall von Arbeitsplätzen bedeutet, ist es in der Radiologie genau gegenteilig. Jedes neue Gerät ist ein neuer Arbeitsplatz.

Insgesamt ist die radiologische Welt heute deutlich komplexer. Wir haben kaum noch Institute, die über eine Dunkelkammer verfügen, vielleicht noch im niedergelassenen, teilradiologischen Bereich. Eine klassische Radiologie, ob ambulant oder stationär, verfügt heute über alle digitalen Modalitäten einschließlich RIS und PACS-Systeme. Bilder werden digital nachbearbeitet und digital archiviert. Im Bereich der Schnittbilddiagnostik sind heute ganz andere Bildrekonstruktionen möglich. Es hat sich damit für MTRA nicht nur das Arbeitsfeld verändert, sondern es sind auch völlig andere Arbeitsläufe entstanden.

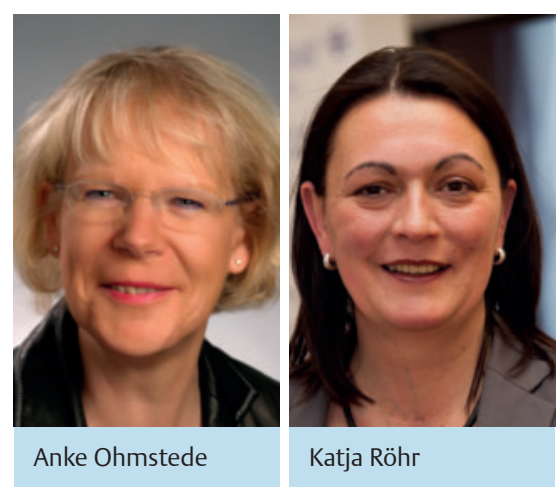

Über die Interviewpartnerinnen

\section{Katja Röhr}

Jahrgang 1965. 1985 Examen an der Berufsfachschule für medizinisch-technische Radiologieassistenten in Zwickau. 1996-1997 Weiterbildung zur Lehrkraft für Diagnostische Radiologie an der Akademie für Lehrkräfte im Gesundheitswesen in Karlsruhe. 1985-1995 als MTRA im Bezirkskrankenhaus Zwickau und Klinikum Nürnberg (Diagnostische Radiologie) tätig. Seit 1995 Fachlehrerin an der MTRA-Schule des Klinikums Nürnberg. Bis 2015 Vorstandsvorsitzende der Vereinigung Medizinisch-Technischer Assistenten in der Deutschen Röntgengesellschaft (VMTB). Seit 2015 kooptiertes Vorstandsmitglied der VMTB.

\section{Anke Ohmstede}

Jahrgang 1953. 1978 MTRA-Examen. Ab 08/1978 als MTRA in den Bereichen Radiologische Diagnostik und Strahlentherapie und ab 1982 in der Nuklearmedizin Klinikum Oldenburg tätig. Seit 1990 Leitung der MTA-Schule Klinikum Oldenburg gGmbH und Lehrerin für Nuklearmedizin, Strahlenschutzrecht und Berufskunde. Von 2000-2004 und seit März 2010 ehrenamtliche Vorstandsvorsitzende des Dachverbands für Technologen/-innen und Analytiker/-innen in der Medizin Deutschland e.V. für die Fachrichtungen Radiologie/Funktionsdiagnostik.

Welche Auswirkungen hat das veränderte Berufsbild auf die bestehenden Ausbildungs- und Prüfungsinhalte?

Röhr: Bei der bestehenden Ausbildungsund Prüfungsverordnung für technische Assistenten in der Medizin von 1994 müssen dringend entsprechende Änderungen 
und Anpassungen vorgenommen werden. Es ist natürlich immer schwierig, alle aktuellen Entwicklungen unterzubringen. Aber es stehen auch Dinge drin, die es schon länger nicht mehr gibt, aber immer noch unterrichtet werden müssen. Dies gilt beispielsweise für die Dunkelkammer oder auch für Film-Folien-Systeme. Das ist, salopp gesagt, Quatsch. Vieles hat nichts mehr mit Grundlagen zu tun, weil es das einfach nicht mehr gibt.

Ohmstede: Wir, DRG, DVTA und VMTB, haben ja bereits gemeinsame Anstrengungen unternommen, erste zaghafte Änderungen in der APrV anzustoßen. Bei Gesprächen mit dem Bundesministerium für Gesundheit im Herbst 2013 konnten wir den akuten Änderungsbedarf überzeugend darstellen. Uns wurde deshalb auch zugestanden, kleine Teile der APrV zu ändern. Hierfür haben wir am 25. Juli 2014 einen Vorschlag eingereicht. Seitdem haben wir leider nichts mehr gehört. Auf Nachfrage Anfang 2015 wurde uns mitgeteilt, dass man den Vorschlag noch bearbeite.

Sie fordern grundsätzlich mehr Handlungs- und Kompetenzorientierung in der Ausbildung. Was genau meinen Sie damit?

Ohmstede: Wir haben zwar eine bundesrechtliche Regelung hinsichtlich der MTAAusbildung, die Durchführung obliegt jedoch den Ländern. Hier in Niedersachsen hat deshalb das zuständige Kultusministerium vor knapp 3 Jahren die Initiative ergriffen und in Zusammenarbeit mit den insgesamt 5 MTA-Schulen eine Rahmenrichtlinie erstellt, die eine Umsetzung von handlungsorientiertem Unterricht zum Ziel hat.

Wir haben den bisherigen Fächerkanon in Handlungsfeldern zusammengeführt und den Unterricht stärker auf die Vermittlung von Kompetenzen zur eigenständigen Problemlösung ausgerichtet. Nehmen Sie das Beispiel Nuklearmedizin. In der technischen Durchführung hat man hier gelegentlich Probleme, die noch nie aufgetreten sind. Wie kann ich diese Probleme analysieren und wie kann ich sie im Sinne des Patienten und des bestmöglichen Ergebnisses lösen? Aus der bisherigen starren Fächersystematik heraus ist es für Schüler und auch für Lehrer sehr schwer, die für die Lösung notwendigen Zusammenhänge herzustellen bzw. didaktisch- methodisch zu vermitteln. Das ist ja im Übrigen auch das, was der Deutsche Qualifikationsrahmen abbildet und was in der beruflichen Bildung heute per Definition maßgeblich ist. Die berufsschulischen dualen Ausbildungen sind heute in ihren Curricula auf Kompetenzen ausgerichtet.

Röhr: In Bayern haben wir keine entsprechende Rahmenrichtlinie wie in Niedersachsen. Hier gelten nach wie vor unser Curriculum und unser Fächerkanon. Es ist vorgegeben, was im 1., 2. bis 6. Semester unterrichtet werden darf. So ist es z.B. nicht möglich, Lerninhalte von einem Jahr in ein anderes zu schieben, was bei manchen Inhalten sicher sinnvoll wäre. So ist erst für das 2. Jahr vorgesehen, dass CT und Schnittbildanatomie vermittelt werden. Die konventionelle Röntgentechnik lernen die Schülerinnen und Schüler dagegen schon im 1. Lehrjahr. Wenn hierbei jedoch dann bspw. ein Fall besprochen wird, der eine Krebs- oder Knochenerkrankung beinhaltet, wäre es durchaus sinnvoll, auch die weiteren Untersuchungsmöglichkeiten, wie CT oder Schnittbildanatomie, zu besprechen. Also fallbasiertes Lernen und nicht an den einzelnen Techniken und Verfahren orientiert, das wäre sehr wünschenswert.

Ohmstede: Das ist in Niedersachsen glücklicherweise anders. Wir bewegen uns jetzt in diesen Lernfeldern. Wir haben bis vor 3 Jahren in der staatlichen Prüfung im schriftlichen Teil nur multiple choiceFragen gestellt. Mittlerweile müssen wir $50 \%$ offene Fragen stellen und möglichst auch fallbasiert. Diese Umstellung erfordert auch von uns Lehrern eine erweiterte Kompetenz im Sinne von Fallformulierung und daraus abzuleitenden Fragen. Jährliche Schulleiterkonferenzen ermöglichen hier einen fachlichen Austausch. Eine Schwierigkeit dabei ist, dass die einzelnen Fächer immer noch identifizierbar sein müssen, hier sind wir an das bundeseinheitliche Prüfungsrecht gebunden.

\section{? Muss eine Novellierung der APrV auch die Einführung einer Ausbil- dungsvergütung einschließen?}

Röhr: Das wäre sicherlich wünschenswert, aber die MTRA-Ausbildung findet an einer Berufsfachschule statt und ist damit eine schulische Ausbildung, für die man BAföG beantragen kann, aber für die es keine Vergütung gibt.
Ohmstede: Wir haben allerdings eine ganze Reihe von Ausbildungen im Gesundheitswesen, die ebenfalls an Berufsfachschulen durchgeführt werden. Diese werden wie die MTRA-Ausbildung über das Krankenhausfinanzierungsgesetz finanziert. Aber interessanterweise gibt es beispielsweise in der Krankenpflege und Kinderkrankenpflege eine Ausbildungsvergütung. Die Auszubildenden in der Pflege werden auch auf den Stellenschlüssel im Krankenhaus angerechnet. Diese Ungleichheit muss man schon hinterfragen. Auch wenn die fehlende Vergütung bei MTRA wahrscheinlich darauf zurückzuführen ist, dass nur 1/3 der Ausbildung in der Routine stattfindet, würde der Beruf mit einer Ausbildungsvergütung sicherlich an Attraktivität gewinnen.

Hat vor diesem Hintergrund das neue Notfallsanitätergesetz von 2014 für Sie Vorbildcharakter?

Ohmstede: Zum gegenwärtigen Zeitpunkt muss man konstatieren, dass das Notfallsanitätergesetz eigentlich das modernste Gesetz ist. Wir haben es hier mit handlungsorientiertem Unterricht zu tun, es gibt Lernfelder, ein Prüfungsrecht, dass sich entsprechend am Handlungsort orientiert. Und wir haben eine 3-jährige Ausbildung, die vergütet wird, was für potenzielle Bewerber attraktiv ist.

Röhr: Gerade die Frage nach einer Vergütung wird ja fast immer von potenziellen Bewerbern gestellt. Viele interessieren sich für den Beruf, nehmen dann aber wieder Abstand oder müssen auch Abstand nehmen, weil sie sich das schlicht nicht leisten können. Wir erleben auch, dass Schüler aus finanziellen Gründen ihre Ausbildung abbrechen müssen. Die fehlende Vergütung ist schon ein großes Problem.

Ohmstede: Darüber besticht das Notfallsanitätergesetz noch durch einen anderen Punkt, nämlich eine Modellklausel, die neben der berufsfachschulischen Ausbildung auch eine hochschulische Ausbildung ermöglicht. Das ist in unserem Gesundheitswesen beispielhaft. Dieses Gesetz bietet in seiner Vielfalt und Durchlässigkeit deutlich mehr Möglichkeiten als das MTA-Gesetz von 1993 bzw. die APrV von 1994. 
? "Radiographer“, „Manipulateur d“Electroradiologie Médicale“ oder "Radiologietechnologe“ - im europäischen Ausland gibt es ganz unterschiedliche Berufsbezeichnungen. Verbirgt sich dahinter auch ein anderes Berufsverständnis, gar eine andere Qualität?

Röhr: Außerhalb Deutschlands hat jedenfalls keiner den Assistenten in seiner Berufsbezeichnung. Auch die Österreicher haben vor 10 Jahren den Weg der Akademisierung beschritten und im Zuge dessen mit dem Radiologietechnologen den Namen entsprechend angepasst. Die Akademisierung kann man durchaus zweigeteilt sehen, aber der Name ist genauso bearbeitungswürdig wie die Ausbildungsund Prüfungsverordnung. Wir sind keine Assistenten, verrichten keine Assistenzarbeit. Wir handeln und arbeiten selbständig. Eine Namensänderung würde sicherlich schon die Attraktivität des Berufs merklich erhöhen.

Ohmstede: Deutschland, Luxemburg und Spanien sind die einzigen Länder in Europa mit einer ausschließlich berufsfachschulischen Ausbildung. Das hat bei uns natürlich mit der besonderen Berufsbildungstradition zu tun, die auch im Ausland hohe Wertschätzung genießt. Mit Bachelor, Master, PhD gibt es inzwischen jedoch auch andere Bildungsabschlüsse im Hochschulbereich und alle europäischen Mitgliedsländer sind auf diesen Zug aufgesprungen. Deutschland ist hier insbesondere im Gesundheitsbereich nur bei ausgewählten Berufen mitgegangen. Der Beruf der/des MTRA zählt leider nicht dazu. Häufig wird die Frage gestellt, ob man denn studiert haben muss, um MTRA zu sein. Auch im Pflegebereich wurde darüber diskutiert, ob jemand, der pflegerische Tätigkeiten am Patienten ausübt, einen Hochschulabschluss haben muss. Aber das ist eine falsche Frage.

Es geht um die Inhalte und hierzu zählen auch die inzwischen sehr komplexen technischen Probleme, die sich aus der zunehmend digitalisierten medizinisch-radiologischen Welt ergeben. Was in der klassischen MTRA-Ausbildung nicht angelegt ist, ist das wissenschaftliche Arbeiten. Das ist auch nicht Gegenstand dieser Ausbildung, das kann sie auch gar nicht in den 3 Jahren vermitteln. Ich bin davon überzeugt, dass wir hochschulisch ausgebildete MTRA brauchen, wie im Sachver- ständigengutachten, im Gutachten vom DKI und auch im Gutachten des Wissenschaftsrates für Gesundheitsfachberufe empfohlen, zu 10-20\%. Im Übrigen werden unsere Berufsabschlüsse ja auch nicht anerkannt. Egal ob in Österreich, England oder den Niederlanden - überall müsste ich noch ein Studium, eine weitere Ausbildung machen. Das ist im Grunde eine Form von Diskriminierung, wenn die deutschen Berufsabschlüsse im Gesundheitswesen im EU-Ausland nur mit einer zusätzlichen Ausbildung anerkannt werden.

\section{? Es gibt ganz offensichtlich einen} unmittelbaren Handlungs- und Änderungsbedarf. Was sind die nächsten Schritte und wie viel Optimismus begleitet Sie bei dem Versuch, die politischen Entscheider auf Ihre Seite zu ziehen?

Röhr: Wir sind in der Pipeline, in der Wartestellung, was sich nicht so toll anfühlt. Natürlich gibt es auch andere wichtige Themen und Anliegen. So haben auch die Zahntechniker einen massiven Überarbeitungsbedarf, zumal deren Ausbildungsund Prüfungsverordnung meines Wissens noch älter ist als unsere. Es wäre trotzdem schon begrüßenswert, wenn zumindest bei kleineren Dingen Fortschritte erreicht werden könnten.

Ohmstede: Die 4 MTA-Berufe stellen mit über 90000 Berufsangehörigen eine große Berufsgruppe dar, die zu einem wesentlichen Teil diagnostische Leistungen erbringt, den Radiologen und Laborfachärzten, aber letzten Endes auch den handelnden Ärzten am Patienten zuarbeitet, deren Therapieerfolg von einer qualitativ hochwertigen Diagnostik abhängt. Alle Welt redet von MINT-Berufen, insbesondere von MINT-Berufen für Frauen. Wir haben hier eine große Berufsgruppe, die MINT ist, die ganz klassisch in den Naturwissenschaften, im Bereich der Technik und in der IT tätig ist. Das wird unverständlicherweise völlig ignoriert. Ich würde mir daher wünschen, dass sich die politischen Entscheider entsprechend der technischen Entwicklung beschleunigen und eine Ausbildungs- und Prüfungsverordnung schaffen, die modern ist.
Perspektiven bietet er heute und in Zukunft?

Röhr: Das Besondere an unserem Beruf ist die Kombination aus Technik und Patientenkontakt. Wir sind kein rein technischer und auch kein rein krankenpflegerischer Beruf, was übrigens auch zunehmend von männlichen Jugendlichen nachgefragt wird.

Ohmstede: Als MTRA trage ich im diagnostischen Sinne dazu bei, dass der Patient behandelt werden kann. Faszinierend ist, wie sich mit der Entwicklung von Soft- und Hardware die medizinische Bildgebung weiter entwickelt hat, wie z.B. die molekulare Bildgebung. D.h., am Ball zu bleiben und sich mit den technischen Anforderungen auseinanderzusetzen, aber dabei den Patienten nicht aus dem Blick zu verlieren. Für die Patienten ist die Komplexität der vielen diagnostischen Möglichkeiten kaum noch nachvollziehbar, insofern sind MTRA wichtige Kommunikatoren zwischen Patient und Technik. Allerdings muss die antiquierte Ausbildungs-und Prüfungsverordnung dringend novelliert werden. Es ist für mich nicht nachvollziehbar, warum wir von der Politik so abgehängt werden.

Vielen Dank für das Gespräch! 